\title{
Certified Reference Material for Strain Measurement Using EBSD
}

M.D. Vaudin ${ }^{1}$, W.A. Osborn ${ }^{1}$, L.H. Friedman ${ }^{1}$ and K. Siebein ${ }^{2}$

1. Materials Measurement Science Division, National Institute of Standards and Technology (NIST), Gaithersburg, MD 20899, USA

${ }^{2}$ Center for Nanoscale Science and Technology, National Institute of Standards and Technology, Gaithersburg, MD 20899, USA

The certified reference material (CRM[1]) for investigating the accuracy of the strain measurement method using electron backscattered diffraction has passed the majority of the tests required before release, and many of these results have recently been described [2]. The method, known as high resolution electron backscattered diffraction (HR-EBSD), measures the strain between positions on a sample in a scanning electron microscope by obtaining EBSD patterns (EBSPs) in a scanning electron microscope (SEM) at those positions and cross correlating several equivalent sub-regions in the patterns; the method has been described by Wilkinson et al. [3], and implemented in both commercial (Crosscourt $[3,4]$ ) and non-commercial (OpenXY[5,6]) software. A reference artifact consists of a $25 \mathrm{~mm}$ square chip cut from an (001)-oriented $\mathrm{Si}$ wafer with a film of $\mathrm{Si}_{1-\mathrm{x}} \mathrm{Ge}_{\mathrm{x}}$ of thickness $(\mathrm{t}$ ) deposited on the wafer by a commercial vendor; the film is patterned and has perfect epitaxy between the film and substrate. The CRM will consist of two chips of $\mathrm{Si}_{-} \mathrm{Si}_{1-\mathrm{x}} \mathrm{Ge}_{\mathrm{x}}$-on-Si with nominal $(\mathrm{t}, \mathrm{x})$ values of $(35 \mathrm{~nm}, 0.3)$ and (50 nm, 0.2), respectively. Since $\mathrm{Si}_{1-\mathrm{x}} \mathrm{Ge}_{\mathrm{x}}$ (shortened where appropriate to $\mathrm{SiGe}$ ) has a larger lattice parameter than $\mathrm{Si}$, the epitaxial $\mathrm{SiGe}$ film is compressed in the film plane and expands normal to the film, producing a tetragonally strained film. NIST reference values for the two tetragonal film strains will be supplied, and values for $t$ and $x$ for both chips will be given as NIST information values. The films are patterned so that among other features at the surface there are stripes of SiGe adjacent to pure $\mathrm{Si}$, with widths from $50 \mu \mathrm{m}$ down to $0.5 \mu \mathrm{m}$. Scanning across these stripes allows EBSPs to be collected from both unstrained and strained material, and pattern analysis software can then be applied to measure the tetragonal strain in the SiGe film. We determine the ratio $(\gamma)$ of the d-spacing of the (001) planes in the SiGe film divided by the (100) and (010) SiGe plane spacings, and the tetragonal strain is defined as $\gamma$-1. The (100) and (010) SiGe plane spacings are assumed to be equal as a consequence of the perfect epitaxy between the $\mathrm{SiGe}$ and $\mathrm{Si}$; this assumption has recently been verified. Another important feature of the film pattern is a $12 \mathrm{~mm} \times 20 \mathrm{~mm}$ pad of SiGe which has been designed for X-ray diffraction (XRD).

The tetragonal strain has been verified using two XRD systems and the degree of epitaxy has been determined by a combination of techniques including XRD and X-ray photoelectron spectroscopy (XPS). Measurements of plane spacings both parallel to the chip surface (symmetric scans) and inclined (asymmetric scans) have been carried out using x-ray reciprocal space mapping around the 004 and 404 diffraction peaks from the substrate and film. Results indicate that for both $(\mathrm{t}, \mathrm{x})$ samples described above, the ratio of the in-plane spacings of the film and substrate are 1.0000(1), indicating perfect filmsubstrate epitaxy. It has also been found the plane spacing ratio for $\mathrm{SiGe}$ and $\mathrm{Si}(004)$ agree within $10^{-4}$ 
with results obtained from symmetric XRD scans measured using a two-circle X-ray diffractometer, and with calculations based on measurements of composition using XPS; many of these results were detailed in [2].

Now that the CRM, consisting of two artifacts with certified tetragonal strain values, has been validated with XRD data as described above, it can function in a way that mirrors the use of standards in energy dispersive spectroscopy (EDS) for quantification of elemental composition in the SEM. In the same way that compositional standards allow the accuracy of EDS in a SEM to be investigated, it becomes possible to assess the accuracy of HR-EBSD, calibrate an EBSD system for accuracy in strain measurement, and develop measurement protocols that maximize strain measurement accuracy. To achieve these goals, EBSPs will be collected at maximum pixel resolution from the same CRM samples using a number of in-house EBSD systems. The data will be collected at different SEM accelerating voltages and working distances to assess the impact of these experimental parameters. Tetragonal strains will be determined using CrossCourt and compared across systems and across experimental and analysis parameters. Initial results have already been collected from two systems and they show that the measured strains from the same sample have a relative difference of up to $10 \%$ and that diffraction patterns from the same system give different results depending on accelerating voltage, working distance and data analysis parameters. It must be noted that the precision of the technique, as indicated by the noise in strain values while scanning across a region of constant strain, can in some cases be as low as $1 \times 10^{-4}$ strain. In a qualitative sense, HR-EBSD when used as a mapping tool can give extremely useful information about strain variation on a sample surface at spatial resolutions at $50 \mathrm{~nm}$ or better, with precision and resolution unobtainable by any other method. Indeed, much of the recent work on improving the technique of HR-EBSD has concentrated on the precision attainable, e.g., [7a,b]. When the strain data are to be compared with other techniques or with HR-EBSD strain data from different systems, potentially from different laboratories, and if the data are to be used in modeling analyses, it is vital to calibrate the results against a known sample such as that provided by the CRM described here.

\section{References:}

[1] NIST Special Publication 260 - 136

[2] MD Vaudin, WA Osborn, LH Friedman, JM Gorham, V Vartanian, RF Cook, Ultramicroscopy 148 (2015), p.94.

[3] AJ Wilkinson, G Meaden, and DJ Dingley, Mater. Sci. Technol. 22 (2006), p. 1271.

[4] Certain commercial equipment, instruments, or materials are identified in this paper to foster understanding. Such identification does not imply recommendation or endorsement by the National Institute of Standards and Technology, nor does it imply that the materials or equipment identified are necessarily the best available for the purpose.

[5] J Kacher, C Landon, BL Adams and D Fullwood, Ultramicroscopy 109(9) (2009), p. 1148.

[6] Brigham Young University (2015). OpenXY V1.0, github.com.

[7] (a) TB Britton, J Jiang, R Clough, E Tarleton, AI Kirkland, AJ Wilkinson, Ultramicroscopy 135 (2013) 126; (b) TB Britton, J Jiang, R Clough, E Tarleton, AI Kirkland, AJ Wilkinson, Ultramicroscopy 135 (2013) 136. 